\title{
On the Mathematical Boundaries of Communication with Zero-Capacity Quantum Channels
}

\author{
Laszlo Gyongyosi ${ }^{1 *}$ and Sandor Imre ${ }^{1}$ \\ ${ }^{1}$ Budapest University of Technology and Economics, Budapest, Hungary \\ gyongyosi@hit.bme.hu
}

\begin{abstract}
In the first decade of the 21 st century, many revolutionary properties of quantum channels were discovered. These phenomena are purely quantum mechanical and completely unimaginable in classical systems. Recently, the most important discovery in Quantum Information Theory was the possibility of transmitting quantum information over zerocapacity quantum channels. In this work we prove that the possibility of superactivation of quantum channel capacities is determined by the mathematical properties of the quantum relative entropy function.
\end{abstract}

\section{Introduction}

The superactivation of quantum channels is an extreme violation of the additivity of quantum channels (Hastings, 2009). This effect makes possible the communication over zero-capacity quantum channels. The superactivation effect was discovered by Smith and Yard in 2008 (Smith and Yard, 2008), who demonstrated that this effect works for the quantum capacity (Smith et al., 2011). Later, these results were extended to the classical zero-error capacity (Cubitt and Smith 2009), (Cubitt et al., 2009) and to the quantum zero-error capacity (Duan, 2009). An algorithmic solution to the problem was developed in (Gyongyosi and Imre, 2012). Currently, we have no theoretical background for describing all possible combinations of superactive zero-capacity channels; hence, there may be many other possible combinations (Gyongyosi and Imre, 2012), (Gyongyosi and Imre, 2012a), (Brandao and Oppenheim, 2010), (Brandao et al, 2011).

In this paper we prove that the problem of superactivation is rooted in information geometric issues and there is a strict connection between the mathematical properties of the quantum relative entropy function and the possibility of superactivation. As we have discovered, the set of superactive channel combinations is limited and determined by the quantum relative entropy function. Before our work this fundamental and purely mathematical connection between the quantum relative entropy function and the superactivation effect was completely unrevealed. We demonstrate the results for the quantum capacity; however the proposed theorems and connections hold for all other channel capacities of quantum channels for which the superactivation is possible (Imre and Gyongyosi, 2012), (Imre and Balazs, 2005). 


\section{The Quantum Capacity of a Quantum Channel}

In this paper, the results are illustrated with the $Q\left(\mathcal{N}_{1} \otimes \mathcal{N}_{2}\right)$ quantum capacity of the joint structure, $\mathcal{N}_{1} \otimes \mathcal{N}_{2}$. The proposed theorems hold for all channel capacities of the joint channel $\mathcal{N}_{1} \otimes \mathcal{N}_{2}$ for which the superactivation is possible. These capacities are the $Q^{(1)}\left(\mathcal{N}_{1} \otimes \mathcal{N}_{2}\right), Q\left(\mathcal{N}_{1} \otimes \mathcal{N}_{2}\right)$, the zeroerror classical capacities $C_{0}^{(1)}\left(\mathcal{N}_{1} \otimes \mathcal{N}_{2}\right), \quad C_{0}\left(\mathcal{N}_{1} \otimes \mathcal{N}_{2}\right)$ and the zero-error quantum capacities $Q_{0}^{(1)}\left(\mathcal{N}_{1} \otimes \mathcal{N}_{2}\right), Q_{0}\left(\mathcal{N}_{1} \otimes \mathcal{N}_{2}\right)$.

The classical and the quantum capacities of quantum channels are described by the HolevoSchumacher-Westmoreland (HSW) (Holevo, 1998), (Schumacher and Westmoreland, 1997) and the LloydShor-Devetak (LSD) (Lloyd, 2009), (Shor, 2002), (Devetak, 2005) theorems (Gyongyosi and Imre, 2011), (Gyongyosi and Imre, 2012), (Imre and Gyongyosi, 2012a). In case of the quantum capacity $Q(\mathcal{N})$, the correlation measure is the quantum coherent information function. The single-use quantum capacity of quantum channel $\mathcal{N}$ is the maximization of the $I_{\text {coh }}$ quantum coherent information:

$$
Q^{(1)}(\mathcal{N})=\max _{\text {all } p_{i}, \rho_{i}} I_{\text {coh }}
$$

The $I_{\text {coh }}\left(\rho_{A}: \mathcal{N}\left(\rho_{A}\right)\right)$ quantum coherent information can be expressed as

$$
\begin{aligned}
I_{\text {coh }}\left(\rho_{A}: \mathcal{N}\left(\rho_{A}\right)\right) & =\mathrm{S}\left(\mathcal{N}\left(\rho_{A}\right)\right)-\mathrm{S}_{E}\left(\rho_{A}: \mathcal{N}\left(\rho_{A}\right)\right) \\
& =\mathrm{S}\left(\rho_{B}\right)-\mathrm{S}\left(\rho_{E}\right),
\end{aligned}
$$

where $\mathrm{S}(\rho)=-\operatorname{Tr}(\rho \log (\rho))$ is the von Neumann entropy and $\mathrm{S}_{E}\left(\rho_{A}: \mathcal{N}\left(\rho_{A}\right)\right)$ is the entropy exchange. In the proof we exploit a connection ${ }^{1}$ between the Holevo information and the quantum coherent information. As it has been shown by Schumacher and Westmoreland (Schumacher and Westmoreland, 2000), the quantum coherent information also can be expressed with the help of Holevo information, as follows

$$
I_{c o h}\left(\rho_{A}: \mathcal{N}\left(\rho_{A}\right)\right)=\left(\mathcal{X}_{A B}-\mathcal{X}_{A E}\right)
$$

where

$$
\mathcal{X}_{A B}=\mathrm{S}\left(\mathcal{N}_{A B}\left(\rho_{A B}\right)\right)-\sum_{i} p_{i} \mathrm{~S}\left(\mathcal{N}_{A B}\left(\rho_{i}\right)\right)
$$

and

$$
\mathcal{X}_{A E}=\mathrm{S}\left(\mathcal{N}_{A E}\left(\rho_{A E}\right)\right)-\sum_{i} p_{i} \mathrm{~S}\left(\mathcal{N}_{A E}\left(\rho_{i}\right)\right)
$$

measure the Holevo quantities between Alice and Bob, and between Alice and environment $E$, where $\rho_{A B}=\sum_{i} p_{i} \rho_{i}$ and $\rho_{A E}=\sum_{i} p_{i} \rho_{i}$ are the average states. As follows, the single-use quantum capacity $Q^{(1)}(\mathcal{N})$ can be expressed as

$$
\begin{aligned}
Q^{(1)}(\mathcal{N})=\max _{\text {all } p_{i}, \rho_{i}}( & \left.\mathcal{X}_{A B}-\mathcal{X}_{A E}\right) \\
= & \max _{\text {all } p_{i}, \rho_{i}} \mathrm{~S}\left(\mathcal{N}_{A B}\left(\sum_{i=1}^{n} p_{i}\left(\rho_{i}\right)\right)\right)-\sum_{i=1}^{n} p_{i} \mathrm{~S}\left(\mathcal{N}_{A B}\left(\rho_{i}\right)\right) \\
& -\mathrm{S}\left(\mathcal{N}_{A E}\left(\sum_{i=1}^{n} p_{i}\left(\rho_{i}\right)\right)\right)+\sum_{i=1}^{n} p_{i} \mathrm{~S}\left(\mathcal{N}_{A E}\left(\rho_{i}\right)\right)
\end{aligned}
$$

\footnotetext{
${ }^{1}$ This connection is a rather surprising but not well known result in Quantum Information Theory, for the details see the proof of Eq. 70 in (Schumacher and Westmoreland, 2000).
} 
where $\mathcal{N}\left(\rho_{i}\right)$ represents the $i$-th output density matrix obtained from the quantum channel input density matrix $\rho_{i}$. The asymptotic quantum capacity $Q(\mathcal{N})$ can be expressed by

$$
\begin{aligned}
& Q(\mathcal{N})=\lim _{n \rightarrow \infty} \frac{1}{n} Q^{(1)}\left(\mathcal{N}^{\otimes n}\right)=\lim _{n \rightarrow \infty} \frac{1}{n \text { all } p_{i}, \rho_{i}} I_{\text {coh }}\left(\rho_{A}: \mathcal{N}^{\otimes n}\left(\rho_{A}\right)\right) \\
&=\lim _{n \rightarrow \infty} \frac{1}{n} \max _{\text {all }}\left(\mathcal{P}_{i}, \rho_{i}\right. \\
&\left(\mathcal{X}_{A B}-\mathcal{X}_{A E}\right)^{\otimes n} .
\end{aligned}
$$

As summarize, the quantum capacity $Q(\mathcal{N})$ of a quantum channel $\mathcal{N}$ can be defined by $\mathcal{X}_{A B}$, the Holevo quantity of Bob's output and by $\mathcal{X}_{A E}$, the information leaked to the environment during the transmission. The quantum relative entropic distance between quantum states $\rho$ and $\sigma$ is defined by the quantum relative entropy function $D(\cdot \| \cdot)$ as

$$
\begin{aligned}
& D(\rho \| \sigma)=\operatorname{Tr}(\rho \log (\rho))-\operatorname{Tr}(\rho \log (\sigma)) \\
& =\operatorname{Tr}[\rho(\log (\rho)-\log (\sigma))] .
\end{aligned}
$$

The Holevo quantity can be expressed by the quantum relative entropy function as (Cortese, 2002), (Cortese, 2003), (Schumacher and Westmoreland, 1999), (Schumacher and Westmoreland, 2000), (Petz and Sudar, 1996), (Petz, 2007), (Petz, 2008),

$$
\chi=D\left(\rho_{k} \| \sigma\right),
$$

where $\rho_{k}$ denotes an optimal (for which the Holevo quantity will be maximal) channel output state and $\sigma=\sum p_{k} \rho_{k}$ is the mixture of the optimal output states (Schumacher and Westmoreland, 1999). The Holevo information $\mathcal{X}$ can be derived in terms of the quantum relative entropy in the following way (Cortese, 2002), (Cortese, 2003), (Schumacher and Westmoreland, 1999), (Schumacher and Westmoreland, 2000),

$$
\begin{aligned}
\sum_{k} p_{k} D\left(\rho_{k} \| \sigma\right) & =\sum_{k}\left(p_{k} \operatorname{Tr}\left(\rho_{k} \log \left(\rho_{k}\right)\right)-p_{k} \operatorname{Tr}\left(\rho_{k} \log (\sigma)\right)\right) \\
& =\sum_{k}\left(p_{k} \operatorname{Tr}\left(\rho_{k} \log \left(\rho_{k}\right)\right)\right)-\operatorname{Tr}\left(\sum_{k}\left(p_{k} \rho_{k} \log (\sigma)\right)\right) \\
& =\sum_{k}\left(p_{k} \operatorname{Tr}\left(\rho_{k} \log \left(\rho_{k}\right)\right)\right)-\operatorname{Tr}(\sigma \log (\sigma)) \\
& =\mathrm{S}(\sigma)-\sum_{k} p_{k} \mathrm{~S}\left(\rho_{k}\right)=\mathcal{X} .
\end{aligned}
$$

We express the Holevo information between Alice and Bob as

$$
\mathcal{X}_{A B}=\mathrm{S}\left(\mathcal{N}_{A B}\left(\sum_{i=1}^{n} p_{i} \rho_{i}\right)\right)-\sum_{i=1}^{n} p_{i} \mathrm{~S}\left(\mathcal{N}_{A B}\left(\rho_{i}\right)\right)=D\left(\rho_{k}^{A B} \| \sigma^{A B}\right) .
$$

The second quantity measures the Holevo information which is leaked to the environment during the transmission as

$$
\mathcal{X}_{A E}=\mathrm{S}\left(\mathcal{N}_{A E}\left(\sum_{i=1}^{n} p_{i} \rho_{i}\right)\right)-\sum_{i=1}^{n} p_{i} \mathrm{~S}\left(\mathcal{N}_{A E}\left(\rho_{i}\right)\right)=D\left(\rho_{k}^{A E} \| \sigma^{A E}\right) .
$$




\subsection{Quantum Relative Entropic Expression}

Using the resulting quantum relative entropy function and the Lloyd-Shor-Devetak (LSD) theorem (Lloyd, 2009), (Shor, 2002), (Devetak, 2005), the asymptotic LSD capacity $Q(\mathcal{N})$ can be expressed with as follows

$$
\begin{aligned}
& Q(\mathcal{N})=\lim _{n \rightarrow \infty} \frac{1}{n} Q^{(1)}\left(\mathcal{N}^{\otimes n}\right) \\
& =\lim _{n \rightarrow \infty} \frac{1}{n} \max _{p_{1}, \ldots, p_{n}, \rho_{1}, \ldots, \rho_{n}} I_{c o h}\left(\rho_{A}: \mathcal{N}^{\otimes n}\left(\rho_{A}\right)\right) \\
& =\lim _{n \rightarrow \infty} \frac{1}{n} \max _{p_{1}, \ldots, p_{n}, \rho_{1}, \ldots, \rho_{n}}\left(\mathcal{X}_{A B}-\mathcal{X}_{A E}\right)^{\otimes n} \\
& =\lim _{n \rightarrow \infty} \frac{1}{n} \max _{p_{1}, \ldots, p_{n}, \rho_{1}, \ldots, \rho_{n}} \mathrm{~S}\left(\mathcal{N}_{A B}^{\otimes n}\left(\sum_{i=1}^{n} p_{i} \rho_{i}\right)\right)-\sum_{i=1}^{n} p_{i} \mathrm{~S}\left(\mathcal{N}_{A B}^{\otimes n}\left(\rho_{i}\right)\right) \\
& =\lim _{n \rightarrow \infty} \frac{1}{n} \sum_{n}\left(\min _{\sigma_{1 . . n}} \max _{\rho_{1 . . n}} D\left(\rho_{k}^{A B} \| \sigma^{A B}\right)-\min _{\sigma_{1 . . . n}} \max _{\rho_{1 . . n}} D\left(\rho_{k}^{A E} \| \sigma^{A E}\right)\right) \\
& =\lim _{n \rightarrow \infty} \frac{1}{n} \sum_{n}\left(\sum_{\sigma_{1 . . . n}}^{\otimes n} \operatorname{mix}_{\rho_{1 . . n}} D\left(p_{i} \rho_{i}\right)\right)+\sum_{i=1}^{n} p_{i} \mathrm{~S}\left(\mathcal{N}_{A E}^{\otimes n}\left(\rho_{i}\right)\right)
\end{aligned}
$$

where $\mathcal{X}_{A B}$ is the Holevo quantity of Bob's output, $\mathcal{X}_{A E}$ is the information leaked to the environment during the transmission, $\rho_{k}^{A B}$ is Bob's optimal output state, $\rho_{k}^{A E}$ is the environment's optimal state, $\sigma^{A B}$ is Bob's optimal output average state, $\sigma^{A E}$ is the environment's average state, while $\rho_{k}^{A B-A E}$ is the final optimal output channel state and $\sigma^{A B-A E}$ is the final output average state. The term $A B-A E$ denotes the information which is transmitted from Alice to Bob minus the information which is leaked to the environment during the transmission. For joint structure $\mathcal{N}_{12}=\mathcal{N}_{1} \otimes \mathcal{N}_{2}$ the single-use joint quantum capacity can be expressed by the $D(\cdot \| \cdot)$ quantum relative entropy function as

$$
\begin{aligned}
& Q^{(1)}\left(\mathcal{N}_{1} \otimes \mathcal{N}_{2}\right) \\
= & \min _{\sigma} \max _{\rho}\left(D\left(\rho_{12}^{A B} \| \sigma_{12}^{A B}\right)-D\left(\rho_{12}^{A E} \| \sigma_{12}^{A E}\right)\right) \\
= & \min _{\sigma} \max _{\rho} D\left(\rho_{12}^{A B-A E} \| \sigma_{12}^{A B-A E}\right),
\end{aligned}
$$

where $\rho_{12}^{A B}$ is the optimal output state of joint channel $\mathcal{N}_{12}$, and $\sigma_{12}^{A B}$ is the average state of joint channel $\mathcal{N}_{12}$ between Alice and Bob. The term $E$ denotes the environment, and $A E$ is the channel between Alice and the environment with the optimal state $\rho_{12}^{A E}$, and average state $\sigma_{12}^{A E}$. The final optimal output channel state is depicted by $\rho_{12}^{A B-A E}$, while $\sigma_{12}^{A B-A E}$ is the final output average state of the channel between Alice and the environment. $Q^{(1)}\left(\mathcal{N}_{12}\right)>0$ only if the $\mathcal{N}_{12}=\mathcal{N}_{1} \otimes \mathcal{N}_{2}$ joint structure is superactive, otherwise $Q^{(1)}\left(\mathcal{N}_{1}\right)=Q^{(1)}\left(\mathcal{N}_{2}\right)=Q^{(1)}\left(\mathcal{N}_{12}\right)=0$. The fact that the superactivated quantum capacity can be described by the joint output states of $\mathcal{N}_{1} \otimes \mathcal{N}_{2}$ is summarized in Theorem 1 . 


\section{Results}

Theorem 1. The superactivation of joint structure $\mathcal{N}_{12}=\mathcal{N}_{1} \otimes \mathcal{N}_{2}$ can be analyzed by the joint average $\sigma_{12}^{A B-A E}$ and joint optimal states $\rho_{12}^{A B-A E}$.

Proof. Here, we show that the difference of the quantum relative entropic quantities in (14) can be positive if and only if the channels in $\mathcal{N}_{12}=\mathcal{N}_{1} \otimes \mathcal{N}_{2}$ can activate each other, i.e., the joint channel structure is superactive. According to (14), the quantum relative entropic distance between the $\sigma_{12}^{A B-A E}$ joint average and the optimal joint state $\rho_{12}^{A B-A E}$ is equal to the $Q^{(1)}\left(\mathcal{N}_{1} \otimes \mathcal{N}_{2}\right)$ joint single-use quantum capacity of $\mathcal{N}_{1} \otimes \mathcal{N}_{2} \cdot Q^{(1)}\left(\mathcal{N}_{1} \otimes \mathcal{N}_{2}\right)$ will not be superactivated if the average output joint state $\sigma_{12}^{A B-A E}$ can be given as a product state $\sigma_{12}^{A B-A E}=\sigma_{1}^{A B-A E} \otimes \sigma_{2}^{A B-A E}$. It also must find the optimal output state $\rho_{12}^{A B-A E}$, which can be given as a product state $\rho_{12}^{A B-A E}=\rho_{1}^{A B-A E} \otimes \rho_{2}^{A B-A E}$, the $Q^{(1)}\left(\mathcal{N}_{1} \otimes \mathcal{N}_{2}\right)$. In other words, if $\sigma_{12}^{A B-A E}$ and $\rho_{12}^{A B-A E}$ can be given in a product state formula (i.e., these states are decomposable), and $Q^{(1)}\left(\mathcal{N}_{1} \otimes \mathcal{N}_{2}\right)$ will be zero and the joint structure $\mathcal{N}_{12}=\mathcal{N}_{1} \otimes \mathcal{N}_{2}$ will not be superactive. If these two states cannot be given in tensor product representations, then strict additivity of individual quantum capacities $Q^{(1)}\left(\mathcal{N}_{1}\right)$ and $Q^{(1)}\left(\mathcal{N}_{2}\right)$ will fail and the channel construction $\mathcal{N}_{12}=\mathcal{N}_{1} \otimes \mathcal{N}_{2}$ will be superactive, which leads to $Q^{(1)}\left(\mathcal{N}_{1} \otimes \mathcal{N}_{2}\right)>0$. If the joint states $\sigma_{12}^{A B-A E}$ and $\rho_{12}^{A B-A E}$ are product states, then $Q^{(1)}\left(\mathcal{N}_{1}\right)=Q^{(1)}\left(\mathcal{N}_{2}\right)=Q^{(1)}\left(\mathcal{N}_{1} \otimes \mathcal{N}_{2}\right)=0$, which concludes the proof of Theorem 1 .

These results from the superactivation of the joint structure $\mathcal{N}_{1} \otimes \mathcal{N}_{2}$ are extended to the properties of the joint optimal and average states in Theorem 2.

Theorem 2. The quantum channels $\mathcal{N}_{1}$ and $\mathcal{N}_{2}$ of the joint structure $\mathcal{N}_{12}$ are superactive if and only if the $\sigma_{12}^{A B-A E}$ joint average state and the $\rho_{12}^{A B-A E}$ joint optimal output state of the joint channel structure are entangled states.

Proof. Using the results derived by Cortese (Cortese, 2002), (Cortese, 2003), and Petz et al. (Petz and Sudar, 1996), (Petz, 2007), (Petz, 2008) and Schumacher and Westmoreland (Schumacher and Westmoreland, 1999), (Schumacher and Westmoreland, 2000) the following statements can be made. The "product state formula" form expresses that the channels $\mathcal{N}_{1}$ and $\mathcal{N}_{2}$ of the joint structure $\mathcal{N}_{12}$ cannot activate each other. We use the minimax criterion for the joint states $\rho_{12}^{A B-A E}$ and $\sigma_{12}^{A B-A E}$ along with (14). If the joint average state and the joint optimal output state are entangled states, then the joint channel structure $\mathcal{N}_{12}$ is superactive and the quantum relative entropic distance between the joint states $\rho_{12}^{A B-A E}$ and $\sigma_{12}^{A B-A E}$ is greater than zero. If the quantum channels $\mathcal{N}_{1}$ and $\mathcal{N}_{2}$ of the joint structure $\mathcal{N}_{12}$ can activate each other, then the informational distance of $\rho_{12}^{A B-A E}$ and $\sigma_{12}^{A B-A E}$ cannot be decomposed in the expression of the quantum relative entropy function (Cortese, 2002), (Cortese, 2003), see (14). We will use again that quantum capacity can be expressed from the Holevo information. If joint states $\rho_{12}^{A B-A E}$ and $\sigma_{12}^{A B-A E}$ of the joint channel $\mathcal{N}_{1} \otimes \mathcal{N}_{2}$ are product states, i.e., $\rho_{12}^{A B-A E}=\rho_{1}^{A B-A E} \otimes \rho_{2}^{A B-A E}$ and $\sigma_{12}^{A B-A E}=\sigma_{1}^{A B-A E} \otimes \sigma_{2}^{A B-A E}$, then the $Q^{(1)}\left(\mathcal{N}_{1} \otimes \mathcal{N}_{2}\right)$ joint capacity will be zero, since the quantum relative entropy function $D(\cdot \| \cdot)$ in (14) can be factorized as follows: 


$$
\begin{aligned}
& =Q^{(1)}\left(\mathcal{N}_{1} \otimes \mathcal{N}_{2}\right)=\min _{\sigma_{12}} \max _{\rho_{12}} D\left(\rho_{12}^{A B} \| \sigma_{12}^{A B}\right)-\min _{\sigma_{12}} \max _{\rho_{12}} D\left(\rho_{12}^{A E} \| \sigma_{12}^{A E}\right)= \\
& =\min _{\sigma_{12}^{A B} \rightarrow A E} \max _{\rho_{12}^{A B}-A E} D\left(\rho_{12}^{A B-A E} \| \sigma_{12}^{A B-A E}\right) \\
& =\min _{\sigma_{12}^{A B}-A E} \max _{\rho_{11}^{A B-A E}} \operatorname{Tr}_{12}\left(\left(\rho_{12}^{A B-A E}\right) \log \left(\rho_{12}^{A B-A E}\right)-\left(\rho_{12}^{A B-A E}\right) \log \left(\sigma_{12}^{A B-A E}\right)\right) \\
& =\min _{\sigma_{1}^{A B-A E}} \min _{\sigma_{2}^{A B-A E}} \max _{\rho_{1}^{A B} \max _{\rho_{2}^{A B-A E}}} \operatorname{Tr}_{12}\left(\begin{array}{l}
\left(\rho_{1}^{A B-A E} \otimes \rho_{2}^{A B-A E}\right) \log \left(\left(\rho_{1}^{A B-A E}\right) \otimes\left(\rho_{2}^{A B-A E}\right)\right) \\
-\left(\left(\rho_{1}^{A B-A E}\right) \otimes\left(\rho_{2}^{A B-A E}\right)\right) \log \left(\sigma_{1}^{A B-A E} \otimes \sigma_{2}^{A B-A E}\right)
\end{array}\right)
\end{aligned}
$$

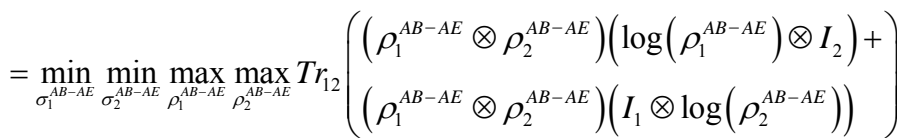

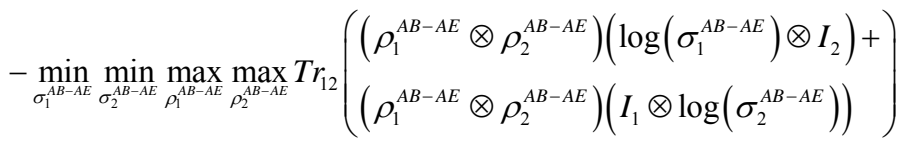

$$
\begin{aligned}
& =\min _{\sigma_{1}^{A B}-A E} \min _{\sigma_{2}^{A B}-A E} \max _{\rho_{1}^{A B-A E}} \max _{\rho_{2}^{A B-A E}} \operatorname{Tr}_{1}\left(\left(\rho_{1}^{A B-A E}\right) \log \left(\rho_{1}^{A B-A E}\right)\right) \operatorname{Tr}_{2}\left(\left(\rho_{2}^{A B-A E}\right) I_{2}\right) \\
& +\min _{\sigma_{1}^{A B-A E}} \min _{\sigma_{2}^{A B-A E}} \max _{\rho_{1}^{A B-A E}} \max _{\rho_{2}^{A B-A E}} \operatorname{Tr}_{1}\left(\left(\rho_{1}^{A B-A E}\right) I_{1}\right) \operatorname{Tr}_{2}\left(\left(\rho_{2}^{A B-A E}\right) \log \left(\rho_{2}^{A B-A E}\right)\right) \\
& -\min _{\sigma_{1}^{A B-A E}} \min _{\sigma_{2}^{A B-A E}} \max _{\rho_{1}^{A B-A E}} \max _{\rho_{2}^{A B-A E}} \operatorname{Tr}_{1}\left(\left(\rho_{1}^{A B-A E}\right) \log \left(\sigma_{1}^{A B-A E}\right)\right) \operatorname{Tr}_{2}\left(\left(\rho_{2}^{A B-A E}\right) I_{2}\right)
\end{aligned}
$$

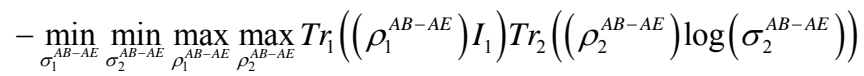

$$
\begin{aligned}
& =\min _{\sigma_{1}^{A B} B A E} \min _{\sigma_{2}^{A B}-A E} \max _{\rho_{1}^{A B}-A E} \max _{\rho_{2}^{A B}-A E}\left(\operatorname{Tr}_{1}\left(\left(\rho_{1}^{A B-A E}\right) \log \left(\rho_{1}^{A B-A E}\right)\right)-\operatorname{Tr}_{1}\left(\left(\rho_{1}^{A B-A E}\right) \log \left(\sigma_{1}^{A B-A E}\right)\right)\right)
\end{aligned}
$$

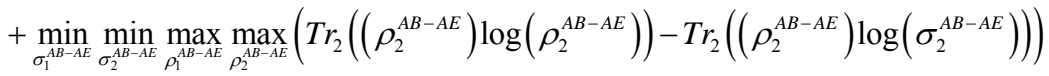

$$
\begin{aligned}
& =\min _{\sigma_{1}^{A B}-A E} \min _{\sigma_{2}^{A B}-A E} \max _{\rho_{1}^{A B}-A E} \max _{\rho_{2}^{A B}}\left(D\left(\rho_{1}^{A B-A E} \| \sigma_{1}^{A B-A E}\right)+D\left(\rho_{2}^{A B-A E} \| \sigma_{2}^{A B-A E}\right)\right)
\end{aligned}
$$

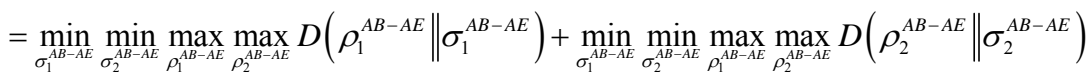

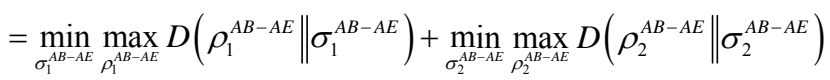

$$
\begin{aligned}
& =Q^{(1)}\left(\mathcal{N}_{1} \otimes \mathcal{N}_{2}\right)=Q^{(1)}\left(\mathcal{N}_{1}\right)+Q^{(1)}\left(\mathcal{N}_{2}\right)=0,
\end{aligned}
$$

where $I_{1}$ and $I_{2}$ are the $d$ dimensional identity matrices ( $d=2$ for the qubit case), $\rho_{12}^{A B}$ is the optimal output state of the joint channel $\mathcal{N}_{12}$ between Alice and Bob, and $\sigma_{12}^{A B}=\sum_{i} p_{i} \rho_{12}^{A B(i)}$ is the average state of the joint channel $\mathcal{N}_{12}$ between Alice and Bob. The term $E$ denotes the environment, $\rho_{12}^{A E}$ is the optimal state of the channel between Alice and the environment, $\sigma_{12}^{A E}=\sum_{i} p_{i} \rho_{12}^{A E(i)}$ is the average state of the channel between Alice and the environment, $\rho_{12}^{A B-A E}$ is the final optimal output channel state, and $\sigma_{12}^{A B-A E}$ is the final output average state of the joint channel $\mathcal{N}_{1} \otimes \mathcal{N}_{2}$. The factorization of (14) implies that the single-use joint quantum capacity $Q^{(1)}\left(\mathcal{N}_{1} \otimes \mathcal{N}_{2}\right)$ can be derived from the strict sum of independent channel quantum capacities $Q^{(1)}\left(\mathcal{N}_{1}\right)$ and $Q^{(1)}\left(\mathcal{N}_{2}\right)$, thus $Q^{(1)}\left(\mathcal{N}_{1}\right)=Q^{(1)}\left(\mathcal{N}_{2}\right)=Q^{(1)}\left(\mathcal{N}_{12}\right)=0$. If the quantum relative entropic distance of the $\sigma_{12}^{A B-A E}$ joint average and $\rho_{12}^{A B-A E}$ joint optimal states of 
$\mathcal{N}_{1} \otimes \mathcal{N}_{2}$ can be factorized, then the joint states $\sigma_{12}$ and $\rho_{12}$ of the joint channel $\mathcal{N}_{12}$ cannot be entangled states; the superactivation of the joint channel structure $\mathcal{N}_{12}$ is possible if and only if the joint states $\rho_{12}^{A B-A E}$ and $\sigma_{12}^{A B-A E}$ of the joint channel $\mathcal{N}_{12}$ are entangled states. The result on the asymptotic quantum capacity of the joint channel $\mathcal{N}_{1} \otimes \mathcal{N}_{2}$ is

$$
\begin{aligned}
& Q\left(\mathcal{N}_{1} \otimes \mathcal{N}_{2}\right) \\
& =\lim _{n \rightarrow \infty} \frac{1}{n} \sum_{n}\left(\min _{\sigma_{1}^{A B-A E}} \min _{\sigma_{2}^{A B-A E}} \max _{\rho_{1}^{A B-A E}} \max _{\rho_{2}^{A B-A E}} D\left(\rho_{1}^{A B-A E} \| \sigma_{1}^{A B-A E}\right)\right) \\
& +\lim _{n \rightarrow \infty} \frac{1}{n} \sum_{n}\left(\min _{\sigma_{1}^{A B-A E}} \min _{\sigma_{2}^{A B} \rightarrow A E} \max _{\rho_{1}^{A B-A E}} \max _{\rho_{2}^{A B-A E}} D\left(\rho_{2}^{A B-A E} \| \sigma_{2}^{A B-A E}\right)\right)
\end{aligned}
$$

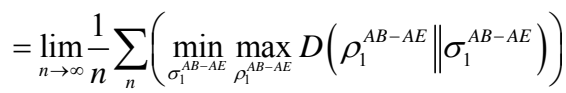

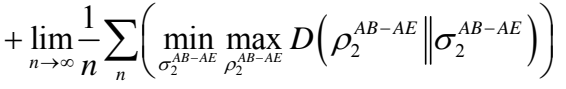

$$
\begin{aligned}
& =Q\left(\mathcal{N}_{1} \otimes \mathcal{N}_{2}\right)=Q\left(\mathcal{N}_{1}\right)+Q\left(\mathcal{N}_{2}\right)=0 .
\end{aligned}
$$

These results conclude the proof of Theorem 2 .

From Theorem 2 also follows that possible set of superactive quantum channels $\mathcal{N}_{1} \otimes \mathcal{N}_{2}$ is also limited by the mathematical properties of the quantum relative entropy function.

\section{Conclusions}

In this paper we proved that the properties of the quantum relative function also determine the superactivation of quantum channels. Our purely mathematical results have demonstrated that the effect of superactivation also depends not only on the channel maps and the properties of the quantum channels of the joint structure as was known before, but on the basic properties of the quantum relative entropy function. Before our work this connection was completely unrevealed in Quantum Information Theory.

\section{Acknowledgment}

The results discussed above are supported by the grant TAMOP-4.2.2.B-10/1--2010-0009 and COST Action MP1006.

\section{References}

Brandao, F.G.S.L. and Oppenheim, J. (2010), Public Quantum Communication and Superactivation, arXiv:1005.1975.

Brandao, F.G.S.L., Oppenheim, J. and S. Strelchuk (2011), When does noise increase the quantum capacity?, arXiv:1107.4385v1 [quant-ph].

Cortese, J. (2002), The Holevo-Schumacher-Westmoreland Channel Capacity for a Class of Qudit Unital Channels", LANL ArXiV e-print quant-ph/0211093. 
Cortese, J. (2003), Classical Communication over Quantum Channels. PhD Thesis by. John A. Cortese. California Institute of Technology.

Cubitt, T. and Smith, G. (2009), Super-Duper-Activation of Quantum Zero-Error Capacities, arXiv:0912.2737v1.

Cubitt, T., Chen, J. X. and Harrow, A. (2009), Superactivation of the Asymptotic Zero-Error Classical Capacity of a Quantum Channel, arXiv: 0906.2547.

Devetak, I. (2005), The private classical capacity and quantum capacity of a quantum channel," IEEE Trans. Inf. Theory, vol. 51, pp. 44-55, quant-ph/0304127.

Duan, R. (2009), Superactivation of zero-error capacity of noisy quantum channels.arXiv:0906.2527.

Gyongyosi, L. and Imre, S. (2011), Information Geometric Superactivation of Classical Zero-Error Capacity of Quantum Channels, Progress in Informatics, Quantum Information Technology, Quantum Information Science Theory Group, National Institute of Informatics, Tokyo, Japan, Print ISSN : 1349-8614, Online ISSN : 1349-8606.

Gyongyosi, L. and Imre, S. (2012), Algorithmic Superactivation of Asymptotic Quantum Capacity of Zero-Capacity Quantum Channels, Information Sciences, ELSEVIER, ISSN: 0020-0255.

Gyongyosi, L. and Imre, S. (2012), Long-Distance Quantum Communications with Superactivated Gaussian Optical Quantum Channels, SPIE Optical Engineering, ISSN: 0091-3286, E-ISSN: 15602303; USA.

Hastings, M. (2009), A Counterexample to Additivity of Minimum Output Entropy" Nature Physics 5, 255, arXiv:0809.3972.

Holevo, A. (1998), The capacity of the quantum channel with general signal states", IEEE Trans. Info. Theory 44, 269 - 273.

Imre, S. and Balazs, F. (2005): Quantum Computing and Communications - An Engineering Approach, Published by John Wiley and Sons Ltd.

Imre, S. and Gyongyosi, L. (2012), Advanced Quantum Communications: An Engineering Approach, Wiley-IEEE Press.

Imre, S. and Gyongyosi, L. (2012), Quantum-assisted and Quantum-based Solutions in Wireless Systems, with Lajos Hanzo, Harald Haas, Dominic O’Brien and Markus Rupp, in: "Wireless Myths, Realities and Futures: From 3G/4G to Optical and Quantum Wireless", Proceedings of the IEEE, ISSN: 0018-9219.

Lloyd, S. (1997), Capacity of the noisy quantum channel, Phys. Rev. A, vol. 55, pp. 1613-1622.

Petz, D. (2007), Bregman divergence as relative operator entropy, Acta Math. Hungar, 116, 127-131.

Petz, D. (2008), Quantum Information Theory and Quantum Statistics: Springer-Verlag, Heidelberg, Hiv: 6.

Petz, D. and Sudar, C. (1996), Geometries of quantum states,” Journal of Mathematical Physics, vol. 37, no. 6, pp. 2662-2673.

Schumacher, B. and Westmoreland, M. (1997), Sending classical information via noisy quantum channels," Phys. Rev. A, vol. 56, no. 1, pp. 131-138.

Schumacher, B. and Westmoreland, M. (1999), Optimal Signal Ensembles, LANL ArXiV e-print quant-ph/9912122.

Schumacher, B. and Westmoreland, M. (2000), Relative Entropy in Quantum Information Theory 2000, LANL ArXiV e-print quant-ph/0004045, to appear in Quantum Computation and Quantum Information: A Millenium Volume, S. Lomonaco, editor (American Mathematical Society Contemporary Mathematics series).

Shor, P. (2002), The quantum channel capacity and coherent information.” lecture notes, MSRI Workshop on Quantum Computation, Available online at http://www.msri.org/publications/ln/msri/2002/quantumcrypto/shor/1/.

Smith, G. and Yard, J. (2008), Quantum Communication with Zero-capacity Channels. Science 321, 1812-1815.

Smith, G., Smolin, J. A. and Yard, J. (2011), Gaussian bosonic synergy: quantum communication via realistic channels of zero quantum capacity. 